Electronic Supplementary Information

\title{
A redox-switchable biocatalyst for controllable oxidation or reduction of 5-hydroxymethylfurfural into high-value derivatives
}

Jiaxing Xu, ${ }^{* \dagger}$ Aiyong He, ${ }^{\dagger}$ Bin Wu,${ }^{\ddagger}$ Lei Hu, ${ }^{* \dagger}$ Xiaoyan Liu,${ }^{\dagger}$ Zhen Wu, ${ }^{\dagger}$ Jun Xia,${ }^{\dagger}$

Jiming Xu, ${ }^{\dagger}$ Shouyong Zhou ${ }^{\dagger}$

$\dagger$ Jiangsu Key Laboratory for Biomass-Based Energy and Enzyme Technology,

Huaiyin Normal University, 111 Changjiangxi Road, Huaian, Jiangsu, 223300, China

\$College of Biotechnology and Pharmaceutical Engineering, Nanjing University of

Technology, 30 Puzhunan Road, Nanjing, 210000, China

\section{Corresponding Authors}

*Jiaxing Xu: Phone: +086051783526983. E-mail: xujiaxing@hytc.edu.cn.

*Lei Hu: Phone: +086051783526983. E-mail: hulei@hytc.edu.cn. 
Table S1 Initial percent transformation of $\mathrm{HMF}$ and $\mathrm{IC}_{50}$ values for $\mathrm{HMF}$

\begin{tabular}{llll}
\hline Strains & Growth $\left(\mathrm{A}_{660}\right)$ & \% Transformation & $\mathrm{IC}_{50}(\mathrm{mM})$ \\
\hline H-2 & 0.68 & 95.9 & 33.3 \\
E-5 & 0.31 & 58.4 & 26.1 \\
G-1 & 0.57 & 82.1 & 29.8 \\
F-18 & 0.94 & 97.2 & 59.2 \\
D-3 & 0.38 & 51.8 & 27.8 \\
K-16 & 0.24 & 39.2 & 14.1 \\
\hline
\end{tabular}

${ }^{\mathrm{a}}$ Initial HMF concentration was $10 \mathrm{mM}$. Glucose served as main substrate. All strains were incubated for $12 \mathrm{~h}$.

Table S2 Strain, plasmids and molecular biological reagent used in the gene engineering.

\begin{tabular}{cc}
\hline Strain and Plasmids & Reagents \\
\hline pKD46 & Pfu polymerase \\
pKD4 & dNTPs、DNA Marker \\
pCP20 & Restriction Enzyme Dpn I \\
E. coli JM109 & DNA Recycling Kit \\
\hline
\end{tabular}

Table S3 Primers used for gene deletion in the gene engineering.

\begin{tabular}{cc}
\hline Primers & Sequence \\
\hline$h m f H-D-R$ & ATGGATCGCTTCGACTACGTGATCGTAGGCGGCGGCTCGGCGGGATGCGTGTAGGCTGGAGCTGCTTC \\
hmfH- $D-F$ & CCGCGACGCCCGAACTCATGCGCGCGGCCATCATGCTCGCGCGGCGTCCATATGAATATCCTCCTTAG \\
hmfH-former & ATGGATCGCTTCGACTACGTGATCGTAGGCGGC \\
hmfH-reverse & CCGCGACGCCCGAACTCATGCGCGCGGCCATCA \\
$P 1$ & GTGTAGGCTGGAGCTGCTTC \\
$P 2$ & CATATGAATATCCTCCTTAG \\
HMFO-D- $R$ & ATGGAGCGCTTCGACTATGTGATCGTCGGCGGCGGGTCCGCGGGCTGCGTGTAGGCTGGAGCTGCTTC \\
HMFO-D-F & CGGCGACGCCGGACGTGAAGCGCGCGGCCGTCATGCTCGCGCGGCGGCCATATGAATATCCTCCTTAG \\
HMFO-former & ATGGAGCGCTTCGACTATGTGATCGTCGGCGGCGG \\
HMFO-reverse & CGGCGACGCCGGACGTGAAGCGCGCGGCCGTCA \\
Verification primer 1 & P1--------hmfH-reverse \\
Verification primer 2 & P1---------hmfO-reverse \\
\hline
\end{tabular}




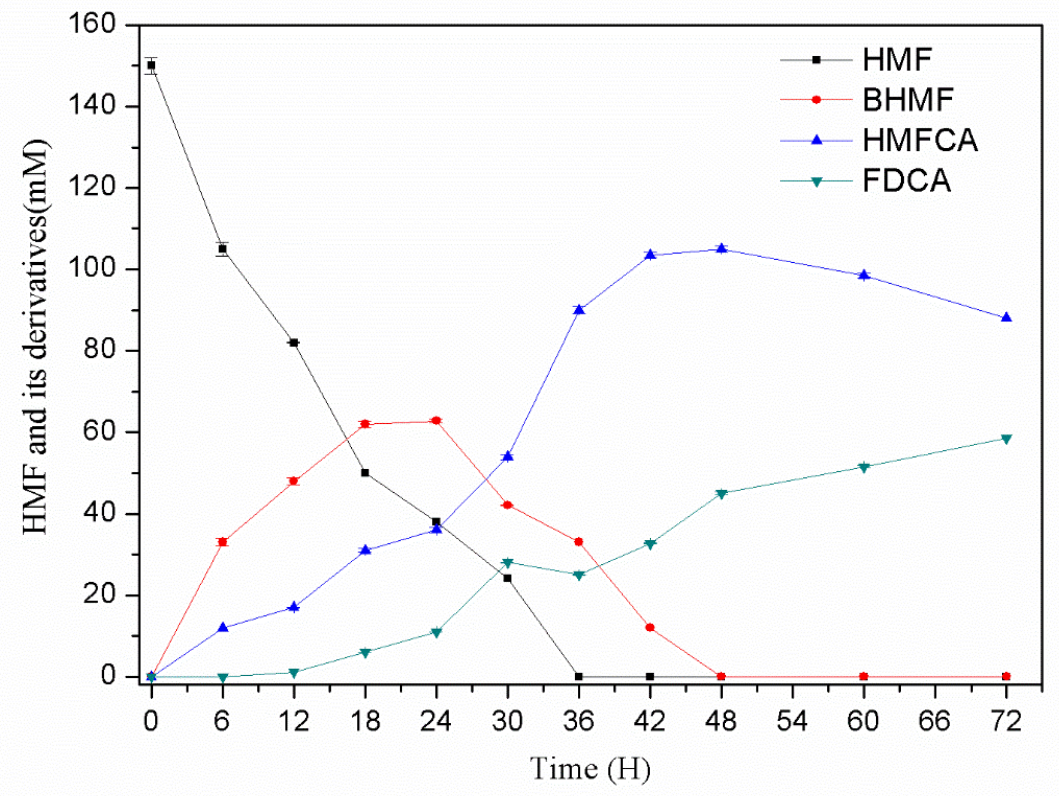

Figure S1 The native transformation ability of $P$. azotifigens $F 18$ toward $150 \mathrm{mM}$ HMF

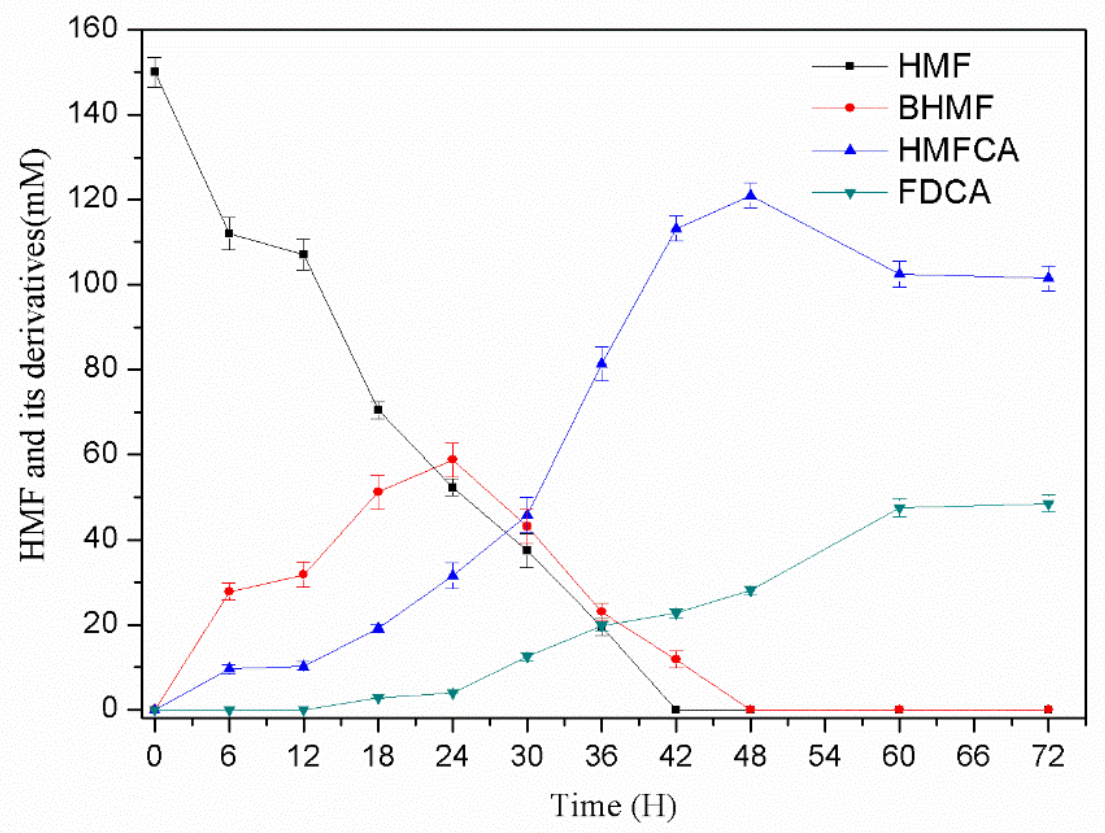

Figure S2 The effect of gene $h m f H$ deletion in $P$. azotifigens F18 on the HMFCA synthesis at a high HMF concentration of $150 \mathrm{mM}$. 


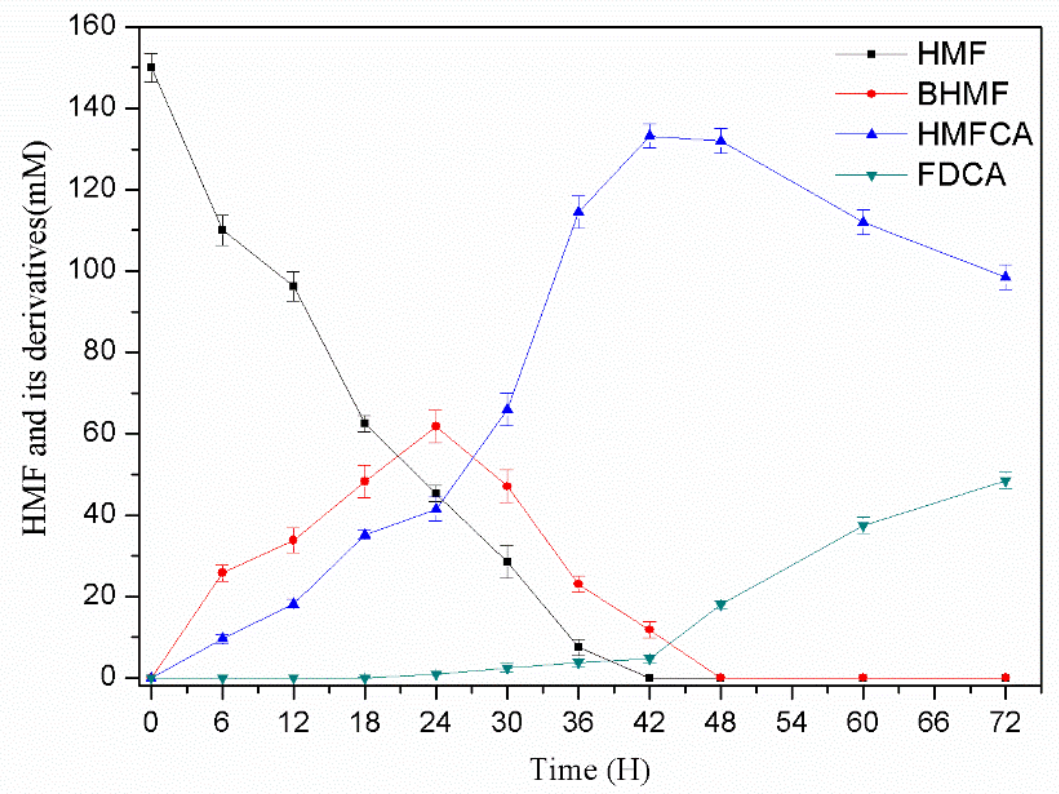

Figure S3 The effect of gene $h m f O$ deletion in P. azotifigens F18 on the HMFCA synthesis at a high HMF concentration of $150 \mathrm{mM}$.

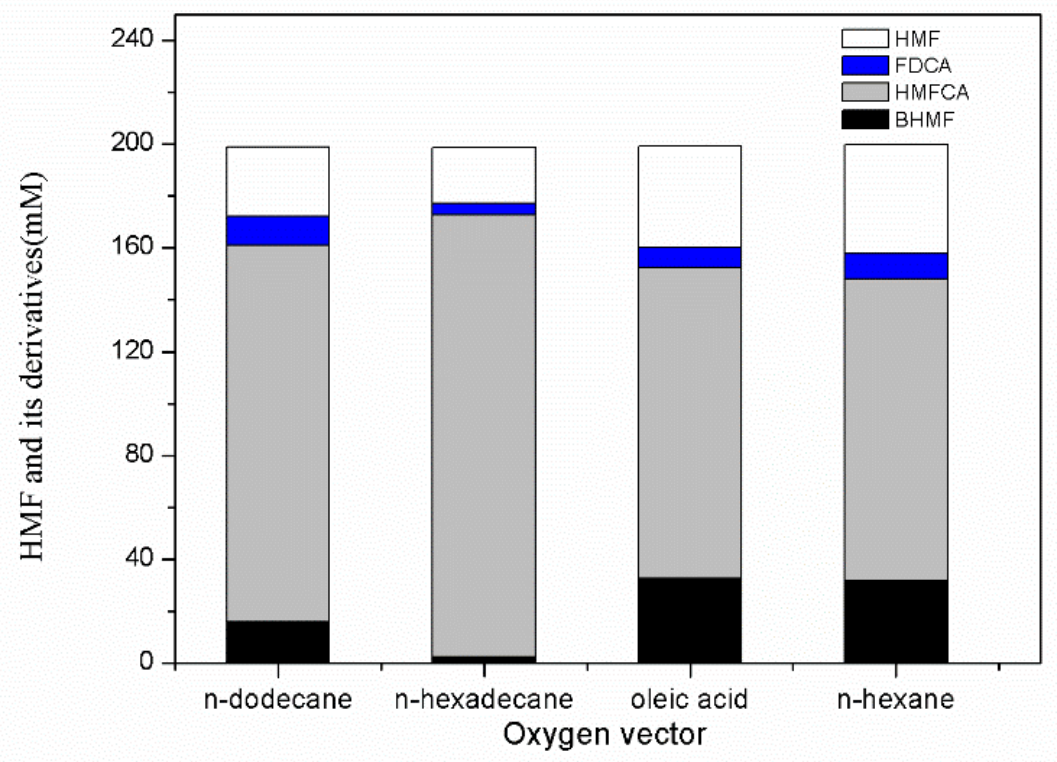

Figure S4 The effect of oxygen vectors on the HMFCA synthesis at a high HMF concentration of $150 \mathrm{mM}$. 


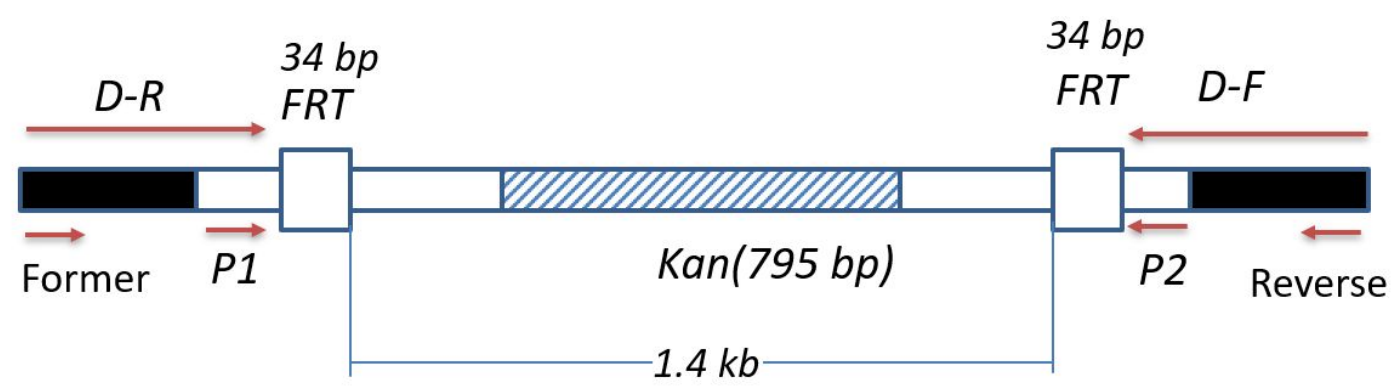

Figure S5 The position of different primers.
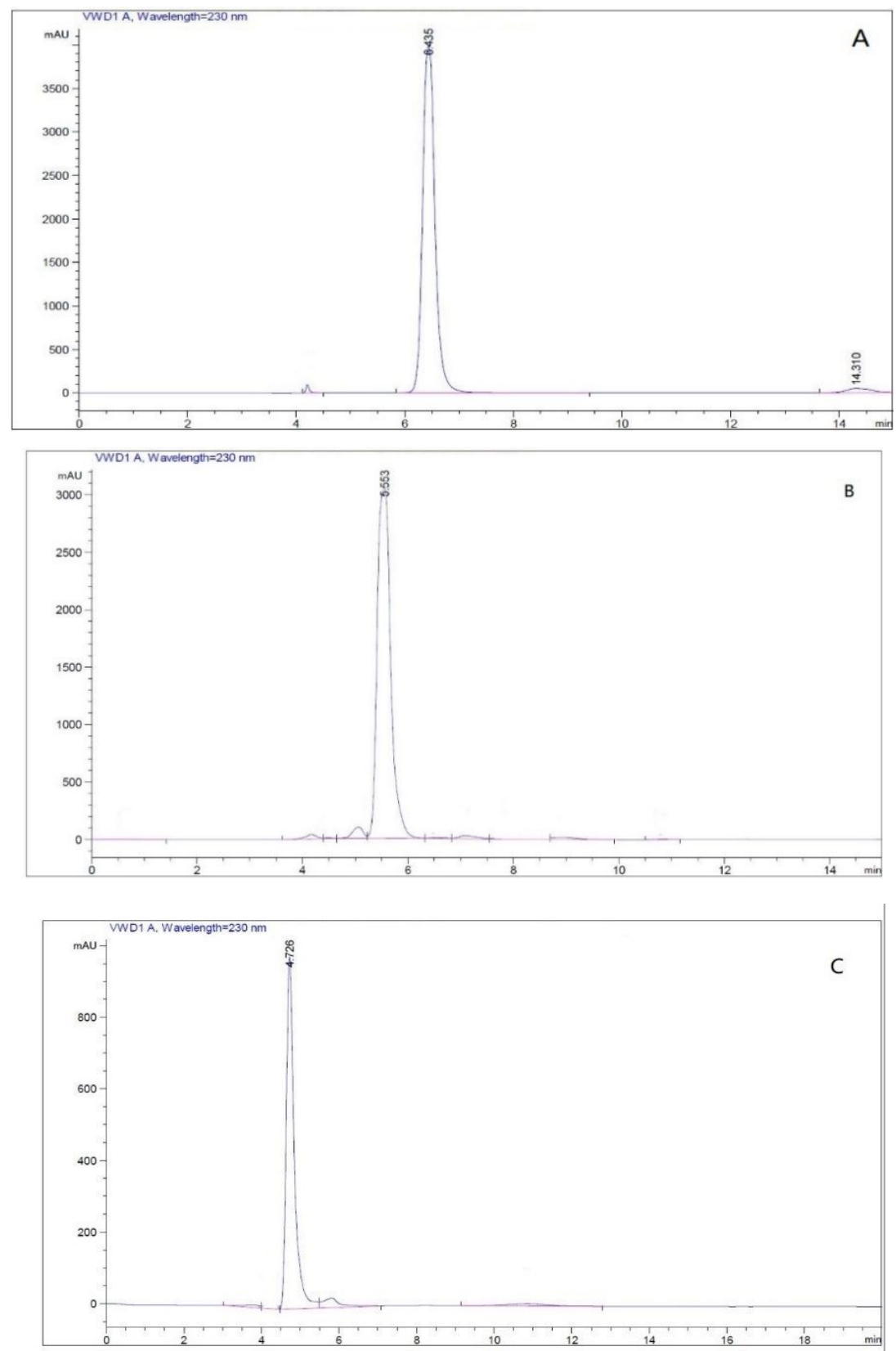


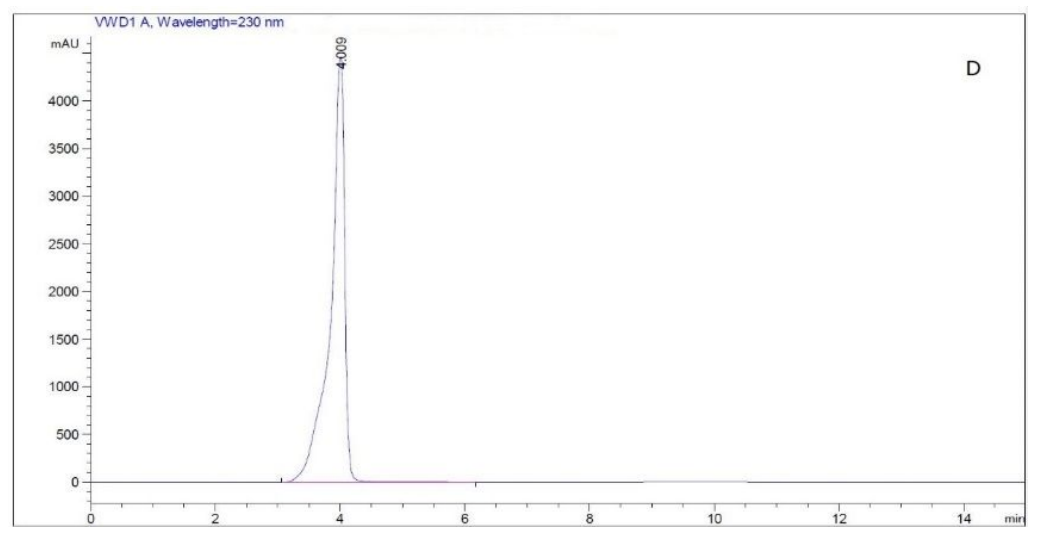

Figure S6 HPLC spectra: (A) HMF, (B) BHMF, (C) HMFCA, (D) FDCA.

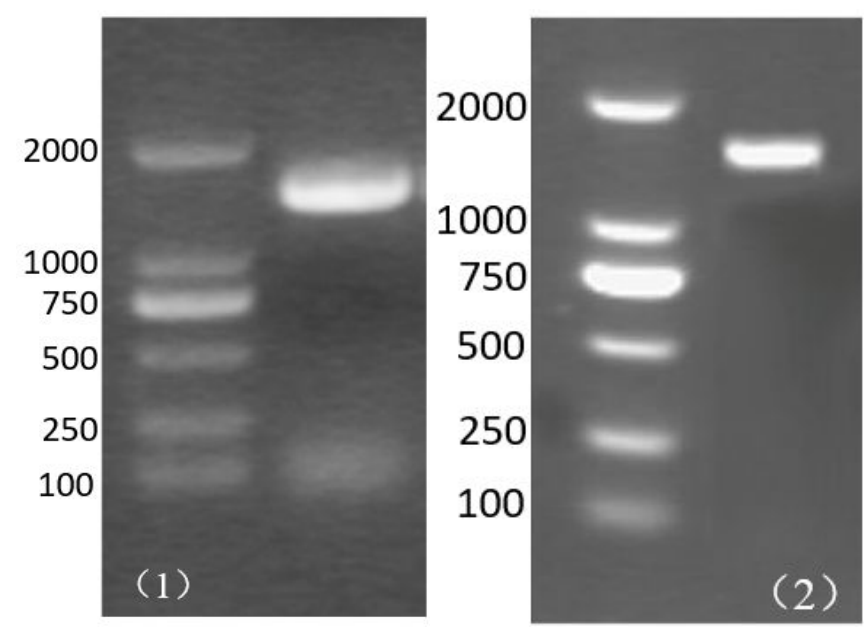

Figure S7 Amplification of homologous fragments for the knockout of gene $h m f H(1)$ and gene $h m f O(2)$.

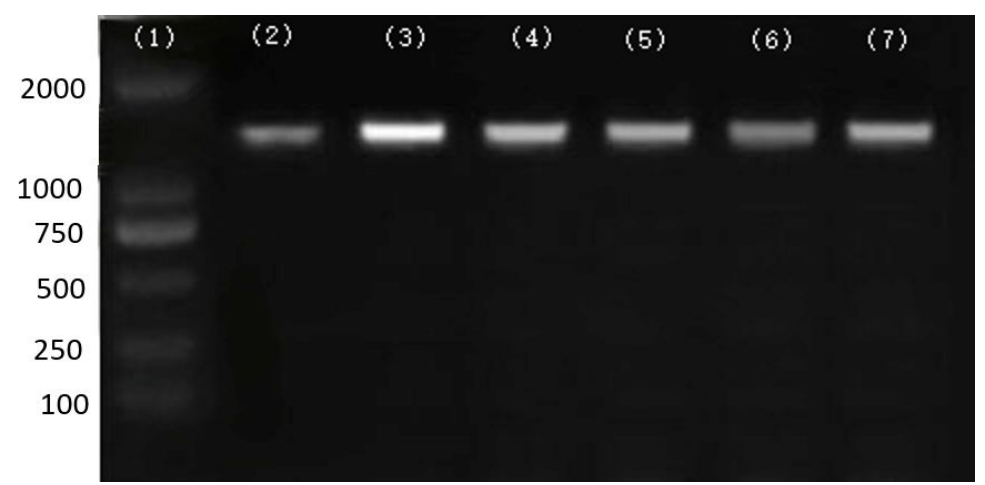

Figure S8 (1) D2000 DNA Marker; Colony PCR verification for the mutant of hmfH (2-4) and $h m f O(5-7)$ using primers for verification as shown in Table S2. 


\section{Sequence of $h m f O$ gene}

ATGGAGCGCTTCGACTATGTGATCGTCGGCGGCGGGTCCGCGGGCTGCGTGCTTGCGCACCGGCTGTCG GCGGTGCCGTCGCTGCGCGTCGCGCTGATCGAGGCCGGCGCGGACACGCCGCCCGGCGCCGTGCCTGCC GAGATTCTCGACAGCTATCCGTTGCCGGTGTTCTGCGGCGACACGTACATCTGGCCGAAGCTGACCGCGC GTGCGACGAAGACCGCCGCGCCGCGCGTATACGAACAGGGCCGCGTGATGGGCGGCGGTTCCAGCGTG AACGTGCAGTCCGCGAACCGCGGGCTGCCGCGCGATTACGACGACTGGGCCGCGAACGGCGCGGCCGG CTGGGCGTGGCAGGACGTGCTGCCGTATTTCCGCAAGCTGGAGCGCGACGTGAATTTTCCGGGCGGCGC GCTGCACGGCAGCGACGGCCCGGTGCCGATTCGCCGGATCATGCCGGACGACTGGCCGCCGTTCTGCCA CGCGTTCGCGGACGGTCTGCGCGCGAACGGCTTCGCGCCATTGCAGGATCAGAACGGCGAGTTCGGCGA CGGCTATTTTCCGGGCGCGTTCTCGAATCTCGACGACCGGCGCGTATCGACCGCCGTCGCGTATCTCGAC GCGGCGACGCGTAGTCGTCCGAATCTGTCGATTTTTCCGCGCCTGCACGTCGAGTCGATCCGCATGGAAG GCGTCGCCGCGCACGGTGTCGTCGCGATCGCGGAGAACGGCGAGCGCGTGACGTTCGACGCGCGCGAA GTGGTGCTCTGCGCCGGCGCGTTGCAGTCGCCCGCGCTGCTGCTGCGCGCGGGCATTGGCCCGCGAGAC GAACTGGCCGCGCTCGGCATCGACTGCCGGCTGGATCTGCCGGGCGTCGGGCGCAATCTGCAGGACCAT CCGTCGCTGACGTTCTGCCATTACCTGGCGCCGCGTTACCGGATGCCGCTGTCGCGCCGCCGCGCGAGCA TGACGGCCGCGCGCTTCACGTCCGGCGTCGCCG

\section{Sequence of $\mathbf{h m f H}$ gene}

ATGGATCGCTTCGACTACGTGATCGTAGGCGGCGGCTCGGCGGGATGCGTGCTTGCGCATCGACTGTCG GCGGAGCCGTCGGTGCGCGTCGCGCTGATCGAGGCGGGCGCCGACACGCCGCCGGGCGCGGTGCCCGC GGCGATTCTCGACAGCTATCCGATGCCCGTCTTCTGCGGCGACACGTACATCTGGCCCGATCTGAAAGCG AAAGCCACGCAGCACGCCGCGCCGCGCGTCTACGAGCAGGGCCGCGTGATGGGCGGCGGGTCGAGCAT CAACGTGCAGTCGGCCAATCGCGGCCTGCCGCGCGACTACGACGAATGGGCCGCGAACGGCGCCGAAG GCTGGTCGTGGCGCGAGGTGCTGCCGTACTTTCGCAAGCTCGAACGCGACGTGAACTTTCCCGGCGGCG AATTGCACGGCAGCGACGGTCCCGTGCCGATCCGCCGCATCATGCCGAACGACTGGCCAGCGTTCTGTCG TGCATTCGCGAAGGGTTTGCGCGCGAACGGCTTCGCGGAACTTCAGGACCAGAACGGCGAATTCGGCGA CGGCTTCTTTCCGGGCGCGTTCTCGAACATCGACGACAAGCGCGTGTCGACGGCCATCGCCTATCTCGAC GAAGCCACGCGCAAGCGCCCGAATCTCAGCATCTATTCGAATCTGCGCGTCGAACGCATCGTGATGGAAG GTGCGACGGCGCGCGGTGTCGTCGCGATTGCGGCGAACGGCGAGCGCGTGCGTTTCGATGCGGGCGAA GTGGTGCTGAGCGCGGGCGCGTTGCAGTCGCCCGCGATGCTGATGCGCGCCGGTATCGGCGACGCGCAC CAACTCGTGGCGATGGGTATCCCGTGTACGGTCGATTTGCCGGGCGTCGGACGCAATCTGCAGGATCATC CGTCGCTCACGTTCTGTCATTTTCTCGAGCCGCGCTTTCGGATGCCGCTCGCGCGACGCCGCGCGAGCATG ATGGCCGCGCGCATGAGTTCGGGCGTCGCGG

\section{Sr DNA sequence of $P$. azotifigens F18}

ACGGTTACCCTGGTCCGACTTCACCCCAATCATGAATCCTAGCGTGGTGACCCTCCTCCTTGCGGTTAGACT AGCCACCTTCTTGGTAAAACCCACCTCCCATGGTGTGACGGGCGGTGTGTACAAGACCCGGGAACGTATT CACCGCGGCATGCTGATCCGCGATTACTAGCGATTCCAGCTTCACGCTGTCGAGTTGCAGACTGCGATCC GGACAACGATCGGTTTTCTGGGATTGGCTCCACCCCGCGGCTTGGCAACACTCTGTTACGACCATTGTACG TACATCTGGCCCGATCTGAAAGCGAAAGCCACGCAGCACGCCGCGCCGCGCGTCTACGAGCAGGGCCGC GTGATGGGCGGCGGGTCGCGCATCAACGTGCAGTCGGCAAATCGCGGCCCCAACATCTCACGACACGAG CTGACGACAGCCATGCAGCACCTGTGTTACGGCTCCCTTTCGGGCACTCCCACCTCTCAGCAGGATTCCGT ACATGTCACGGGTAGGTAAGGTTTTTCGCGTTGCATCGAATTAATCCACATCATCCACCGCTTCTGCGGGT CCCCGTCAATTCCTTTGAGTTTTAATCTTGCGACCGTACTCCCCAGGCGGTCAACTTCACCCGTTAGCTACG TTACCCAGTCAATGAAGACCCGACAACTAGTTGACATCGTTTAGGGCGAGGACTACCAGGGTATCTAATC CTGTTTGCTCCCCACGCTTTCGTGCATGAGCGTCAGTATTGGCCCAGGGGGCTGCCTTCACCATCGGTATT 
CATCCACATCTCTACGCAATTCACTGCTACACGTGGAATTCTACAATCTGCGCGTCGAACGCATCGTGATG GAAGGTGCGACGGCGCGCGGTGTCGTCGCGATTGCGGCGAACGGCGAGCGCGTGCGTTTCGATGCGGG CGAAGTGGTGCTGAGCGCGGGCGCGCCGCGGCCACTGGCACGTAGTTAGCAGGTGCTTATTCTTCCGGA ACCGTCATCCTCCATCCGTATTAGGGACGAAGTTTTCTATCCGGACAAAAGTGCTTTACAACCCGAAAGCC TAAATCACACACGCGGCATTGCTGGATCAGGGTTGAAACCATTGTCCAAAATTCCCCACTGCTGCAAACCG TAGGAGTCTGGGCCGTGTCTCAGTCCCAGTGTGGATGGTCGTCCTCTCAGACCAGCTACAGATCGTCGCC TTGGTAGGCCTTTACCCCACCAACTAGCTAATCTGCCATCGGCCGCCCCTTGAGCGCGAGGTCCGAAGATC CCCCGCTTTCCTCTCCCGAGCGTAACTCGGGTGCGTGAAGCTGGAATCGCTAGTAATCGCGGATCAGCAT GCCGCGGTGAATACGTTCCCGGGTCTTGTACACACCGCCCGTCACACCATGGGAGTGGGTTTTACCAGAA GTG 